\title{
Surface-enhanced Raman spectroscopy before radical prostatectomy predicts biochemical recurrence better than CAPRA-S
}

This article was published in the following Dove Medical Press journal: International Journal of Nanomedicine

Jiahua Pan, ${ }^{1, *}$ Xiaoguang Shao, ${ }^{1, *}$ Yinjie Zhu, ${ }^{1, *}$ Baijun Dong,' Yanqing Wang,' Xiaonan Kang, ${ }^{2} \mathrm{Na}$ Chen, ${ }^{3}$ Zhenyi Chen, ${ }^{3}$ Shupeng Liu, ${ }^{3}$ Wei Xue'

'Department of Urology, Ren Ji Hospital, School of Medicine, Shanghai Jiao Tong University, Shanghai 200 I27, People's Republic of China; ${ }^{2}$ Department of Biobank, Ren Ji Hospital, School of Medicine, Shanghai Jiao Tong University, Shanghai 200 I27, People's Republic of China; ${ }^{3}$ Shanghai Institute for Advanced Communication and Data Science, Key Laboratory of Specialty Fiber Optics and Optical Access Networks, School of Communication and Information Engineering, Shanghai University, Shanghai, People's Republic of China

*These authors contributed equally to this work

Correspondence: Wei Xue

Department of Urology, Ren Ji Hospital, School of Medicine, Shanghai Jiao Tong University, No 1630 Dongfang Road, Shanghai 200I27, People's Republic of China

Tel +86 02I 68383375

Email uroxuewei@I63.com

Shupeng Liu

Shanghai Institute for Advanced

Communication and Data Science, Key Laboratory of Specialty Fiber Optics and Optical Access Networks, School of Communication and Information Engineering, Shanghai University, 99 Shangda Road, Shanghai, People's Republic of China

Email liusp@shu.edu.cn
Objective: The objective of this study was to evaluate the performance of surface-enhanced Raman spectroscopy (SERS) in the prediction of early biochemical recurrence after radical prostatectomy (RP).

Patients and methods: We synthesized monodisperse gold nanoparticles as SERS-enhanced substrates and analyzed preoperative plasma samples of patients who underwent RP. The roles of clinical risk model (Cancer of the Prostate Risk Assessment [CAPRA] score) and distinctive SERS spectra on prediction of early biochemical recurrence were evaluated. The principal component analysis and linear discriminant analysis (PCA-LDA) were used to manage the spectral data and develop diagnostic algorithm.

Results: A total of 306 preoperative plasma Raman spectra from 102 patients were collected. SERS spectrum from those who developed early biochemical recurrence were compared to those who remained biochemical recurrence-free. The SERS detected more abundant circulating free nucleic acid bases in biochemical recurrence population, presenting significant stronger intensities at SERS spectral bands 725 and $1,328 \mathrm{~cm}^{-1}$. The addition of Raman spectral peak $1,328 \mathrm{~cm}^{-1}$ to CAPRA postsurgical (CAPRA-S) score significantly improved the predictive power of logistic regression model compared to simple CAPRA score $(P<0.001)$. Meanwhile, the leave-one-out cross-validation method was used to validate the PCA-LDA model and revealed the sensitivity, specificity, and accuracy of $65.8 \%, 87.5 \%$, and $79.4 \%$, respectively. The receiver operating characteristic (ROC) curve was used to evaluate the performance of different models. Area under the ROC curve of the CAPRA-S score model alone was 0.77 , however, when combined with Raman spectral peak $1,328 \mathrm{~cm}^{-1}$, it improved to 0.81 .

Conclusion: Our primary results suggested that SERS could be a meaningful technique for prediction of early biochemical recurrence in prostate cancer.

Keywords: surface-enhanced Raman spectroscopy, prostate cancer, radical prostatectomy, early biochemical recurrence

\section{Introduction}

Prostate cancer $(\mathrm{PCa})$ remains the second most common malignancy diagnosed in men worldwide. ${ }^{1}$ About $30 \%$ of the patients who received radical prostatectomy (RP) will develop biochemical recurrence within 5 years. ${ }^{2}$ Previous studies indicated that time to biochemical recurrence was a significant risk factor of cancer progression. Men who experienced early biochemical recurrence (at $\leq 2$ years of RP) were more likely to suffer clinical metastases and cancer-specific mortality. ${ }^{3,4}$ Since patients with high risk cancer progression may benefit from early postoperative androgen blockade or external beam radiation, it could be meaningful to identify those who have high risk 
of early biochemical recurrence., ${ }^{5,6}$ Various models based on patients' clinical and pathologic parameters have been developed for predicting disease recurrence. Among them, the Cancer of the Prostate Risk Assessment (CAPRA) score is widely validated and considered as the best performing tool for disease outcome prediction after RP. ${ }^{7,8}$ The potential predictors of early biochemical recurrence such as Gleason score, tumor stage, and prostate-specific antigen (PSA) level were reported to be not effective enough for patients and physicians to make adjuvant therapy decision..$^{9,10}$

Evidences suggested that PCa has a unique metabolism during the cancer development and progression. Therefore, the circulating metabolic biomolecules could be potential source of diagnostic and prognostic biomarkers for PCa. ${ }^{11}$ Metabolomics studies demonstrated that various components in blood and tissue were significantly different between recurrent and non-recurrent $\mathrm{PCa}$ cases, including phosphorylcholine, glutamate, cysteine, and lipids. ${ }^{12,13}$ In addition, a study implied that preoperative urine and serum metabolite profiling could supplement clinical variables in predicting early biochemical recurrence after RP, suggesting that it could be meaningful to extract component information in preoperative blood for disease recurrence prediction. ${ }^{13,14}$ However, the conventional methods in molecule analysis including mass spectrometry, proton nuclear magnetic resonance spectroscopy (1H-NMR), ELISA, which, more or less, were cumbersome in sample preprocessing, sophisticated in data analysis, and costly in reagent kits.

Surface-enhanced Raman spectroscopy (SERS) might be a suitable technology for analyzing blood components due to its capacity for substance recognition at the molecular level, with quick response, high accuracy and sensitivity, as well as minimal sample requirement. ${ }^{15}$ In the present study, we performed SERS analysis of preoperative plasma and evaluated its predictive value of early biochemical recurrence (at $\leq 2$ years after RP). The performance of distinct Raman spectral peaks was compared to a validated clinical model and CAPRA postsurgical (CAPRA-S) score, and the area under the receiver operating characteristic (ROC) curve was calculated when Raman peaks were added to the CAPRA-S model.

\section{Patients and methods Patients and samples collection}

From January 2013 to May 2015, 102 patients were included in this study. Patients of early biochemical recurrence were identified as those who developed PSA relapse at $\leq 2$ years after $R P$ (PSA $\geq 0.2 \mathrm{ng} / \mathrm{mL}$, confirmed at least once 2 weeks later). Patients of biochemical recurrence free survival (bRFS) were defined as those who maintained serum PSA $<0.2 \mathrm{ng} / \mathrm{mL}$ for $>2$ years. Inclusion criteria were as follows: 1) clinically localized PCa subjected to RP, 2) without neoadjuvant treatment before RP, 3) without adjuvant treatment after RP, and 4) followed until biochemical recurrence or for up to 2 years after RP. Detailed clinical information of all subjects and the preoperative blood sample were collected after the informed consent was signed. This study was conducted in accordance with the Declaration of Helsinki and the experimental protocol was approved by the Institutional Ethics Committee of RenJi Hospital affiliated to Shanghai Jiao Tong University, School of Medicine (approval no Renji[2013]126).

CAPRA-S scores of the two groups (biochemical recurrence group and bRFS group) were calculated and patients were classified as low risk (CAPRA-S 0-2), intermediate risk (CAPRA-S 3-5), and high risk (CAPRA-S 6-10) as previously defined. ${ }^{8}$ Blood samples were previously collected before RP. After 12 hours of overnight fasting, $5 \mathrm{~mL}$ of blood samples was collected from the subjects between 6:00 and 7:00 am with the use of an anticoagulant (EDTA) and was then transported to the biobank in RenJi Hospital. Plasma was obtained by centrifugation at 3,000 rpm for 10 minutes and stored at $-80^{\circ} \mathrm{C}$ until SERS analysis.

\section{Gold nanoparticles (Au-NPs) synthesis and SERS measurement}

Monodisperse citra-stabilized Au-NPs with a quasi-spherical shape of $60 \mathrm{~nm}$ were synthesized following a kinetically controlled seeded growth strategy via the reduction of $\mathrm{HAuCl}_{4}$ by sodium citrate. ${ }^{16}$ The Au-NP solution was concentrated by centrifugation at 10,000 rpm for 10 minutes, and the final concentration was obtained for SERS measurement.

Prior to SERS measurement, $10 \mu \mathrm{L}$ of plasma was homogenously mixed with $20 \mu \mathrm{L}$ of prepared Au-NPs, and the mixture was then kept incubated at $4^{\circ} \mathrm{C}$ for 3 hours. A droplet $(3 \mu \mathrm{L})$ of the resulting mixture was transferred onto a silicon plate for SERS measurement. Renishaw Raman microscope (inVia ${ }^{\mathrm{TM}}$; Gloucestershire, UK) was used for the collection of SERS spectra with a spectral resolution of $\sim 1 \mathrm{~cm}^{-1}$. Samples were excited with a $633-\mathrm{nm} \mathrm{He}-\mathrm{Ne}$ laser with a power of $50 \mathrm{~mW}$. The spectral data acquisition time was 1 second using a Leica DM2500 microscope equipped with an objective lens of L50 (N.A. 0.5) and the spectrum was acquired using WiRE 3.2 software over a wave number range of $400-1,800 \mathrm{~cm}^{-1}$. To collect comprehensive information on the samples, three SERS spectra from different areas of each sample were collected. 


\section{Statistical analysis}

Fluorescence background in the spectra data was removed using a Vancouver Raman algorithm based on the fifthorder polynomial fitting method. Patients' clinical variables and spectra data were compared between two groups of patients using Mann-Whitney $U$ test, independent-sample test, and chi-squared test appropriately. The relationship between Raman peak 1,328 $\mathrm{cm}^{-1}$ and early biochemical recurrence was assessed using Cox regression analysis and Kaplan-Meier analysis.

Principal component analysis and linear discriminant analysis (PCA-LDA) was used to analyze the spectra data and developed diagnostic algorithm. The PCA-LDA model was then validated using the leave-one-out cross-validation method. The ROC curve was generated for Raman spectral peak, CAPRA-S score, adjusted model combined with CAPRA-S score and Raman spectral peak, and the PCA-LDA model based on Raman spectra. The corresponding area under the curve (AUC) was calculated. All statistical analyses were performed using the R-statistical package (R Foundation for Statistical Computing, Vienna, Austria), with $P<0.05$ as statistically significant.

\section{Results}

A total of 102 patients were included in this study. Overall, 38 patients developed early biochemical recurrence with a median time to recurrence of 10.6 months, and 64 patients remained bRFS with a median follow-up time of 42.8 months. As shown in Table S1, compared to bRFS group, the biochemical recurrence group had significantly higher preoperative serum PSA $(P=0.001)$, higher proportion of positive surgical margins $(P=0.004)$, higher pathologic Gleason score $(P=0.011)$, and more advanced clinical stage $(P=0.019)$. Moreover, the CAPRA score was asymmetrically distributed. In the bRFS group, $37.5 \%$ of the men were low risk (CAPRA-S 0-2) and 3\% were high risk (CAPRA-S 6-10), while in the biochemical recurrence patient group, $10.5 \%$ of the men were low risk (CAPRA-S 0-2) and $47 \%$ were high risk (CAPRA-S 6-10).

Figure 1A displays a schematic diagram of SERS monitor system. Figure 1B shows the inelastic scattering mechanism of SERS. Components in blood sample were absorbed onto surface of Au-NPs and excited by laser, then Raman spectra were scattered from chemical bonds within molecules and collected by Raman monitor system. Figure 1C is the transmission electron microscopy image of Au-NPs, which shows the substance bases of SERS in microcosmic perspective. The Au-NPs show homogeneous quasi-spherical shape, with diameter of $60 \pm 10 \mathrm{~nm}$, which can amplify the Raman signal of molecules because of the local electromagnetic fields on metal surfaces.

\section{SERS spectra features of the plasma samples}

The mean plasma Raman spectra of the early biochemical recurrence patients (red line) and bRFS patients (cyan line) are shown in Figure 2A. In all patients, the holistic Raman spectral features were demonstrated in the following peaks with tentative biochemical assignments (Table 1): ${ }^{19-23} 638 \mathrm{~cm}^{-1}(\mathrm{C}-\mathrm{S}$ twist/L-tyrosine); $725 \mathrm{~cm}^{-1}$ (CH bending vibration in adenine, hypoxanthine, and coenzyme $\mathrm{A}) ; 810 \mathrm{~cm}^{-1}(\mathrm{C}-\mathrm{C}-\mathrm{O}$ stretching vibration/L-serine); $1,014 \mathrm{~cm}^{-1}(\mathrm{C}-\mathrm{H}$ stretching vibration/phenylalanine); $1,130 \mathrm{~cm}^{-1}$ (C-N stretching vibration/D-mannose); $1,328 \mathrm{~cm}^{-1}\left(\mathrm{CH}_{2}\right.$ twist vibration/lipids and $\mathrm{C}-\mathrm{H}$ vibration in DNA/RNA); $1,584 \mathrm{~cm}^{-1}(\mathrm{C}=\mathrm{C}$ bending/acetoacetate $)$; and $1,660 \mathrm{~cm}^{-1}(\mathrm{C}=\mathrm{C}$ stretching vibration/amide $\mathrm{I})$. When comparing the plasma Raman spectra between the two groups, the patients of early biochemical recurrence had shown stronger intensities at $725(P<0.01), 1,328(P<0.01)$, and $1,447 \mathrm{~cm}^{-1}$ $(P<0.01)$, while lower intensity at $1,014 \mathrm{~cm}^{-1}(P<0.01)$ (Figure 2B). According to the biochemical assignments of Raman spectra peaks, blood of patients who developed early biochemical recurrence might contain higher concentrations of adenine, hypoxanthine, and coenzyme $\mathrm{A}\left(725 \mathrm{~cm}^{-1}\right)$, lipids and DNA/RNA bases $\left(1,328 \mathrm{~cm}^{-1}\right)$, and collagen and phospholipids $\left(1,447 \mathrm{~cm}^{-1}\right)$, while lower concentrations of phenylalanine $\left(1,014 \mathrm{~cm}^{-1}\right)$ than those who remained bRFS.

\section{Evaluation of Raman spectra for the prediction of early biochemical recurrence}

Cox regression proportional hazard analysis was utilized to evaluate the prognostic value of Raman spectra for early biochemical recurrence. As shown in Table 2, the intensities of Raman peak $1,328 \mathrm{~cm}^{-1}$ were associated with risk of early biochemical recurrence (HR: 1.97, 1.41-2.74, $95 \% \mathrm{CI}, P<0.001$, when $1,328 \mathrm{~cm}^{-1}$ increase by a quarter), and remained significantly associated after adjusting for the CAPRA-S score (HR 1.67, 1.19-2.33, 95\% CI, $P=0.003$ ) in multivariate model. The relevance of Raman peak $1,328 \mathrm{~cm}^{-1}$ to early biochemical recurrence status was validated in Kaplan-Meier curve (Figure 3). Patients with high intensity ( $\geq$ median intensity) in Raman peak $1,328 \mathrm{~cm}^{-1}$ were more likely to develop early biochemical recurrence than those with low intensity ( $<$ median intensity) ( $54.9 \%$ vs $19.6 \%, P<0.01)$.

Meanwhile, we used PCA to analyze these spectra and extracted the first 13 principal components, which accounted 
A

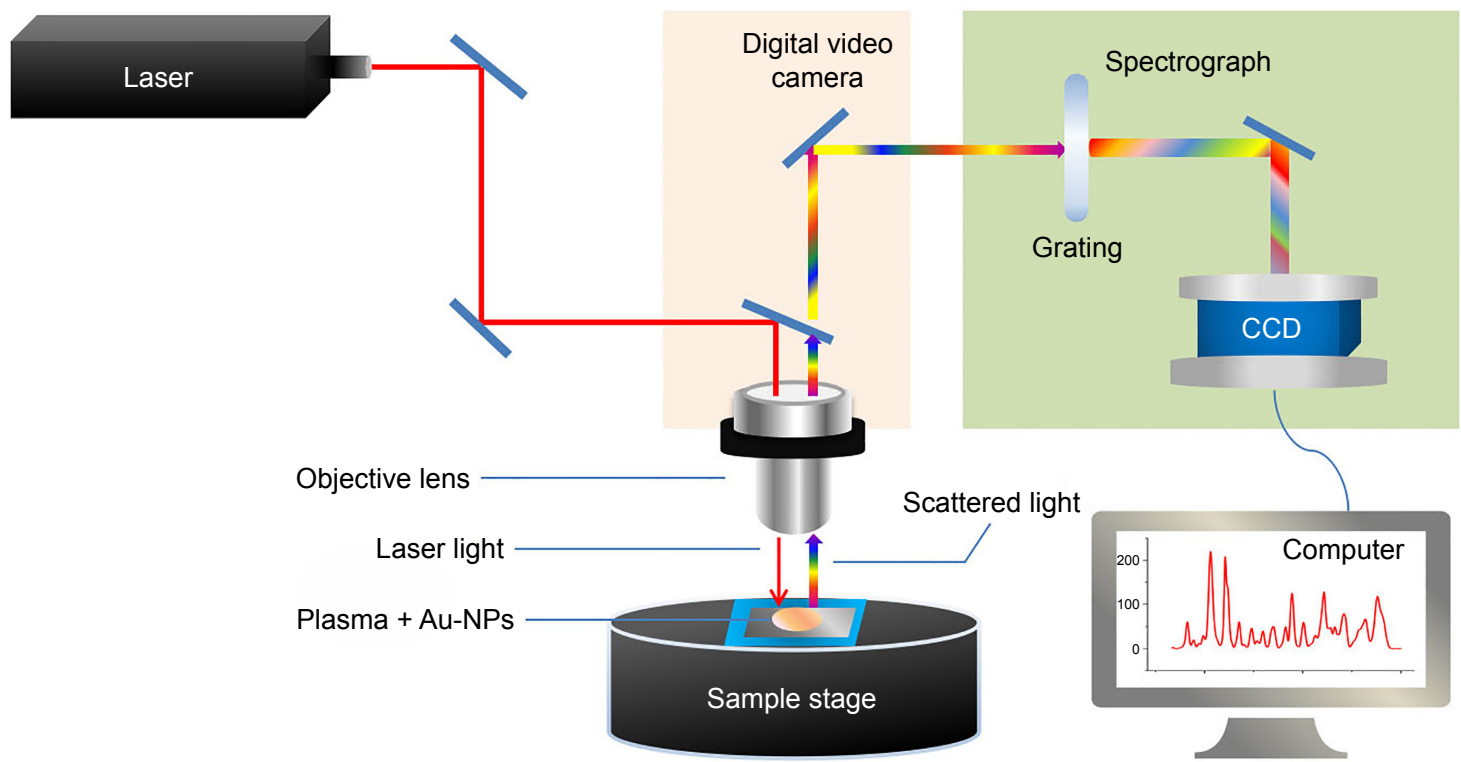

B

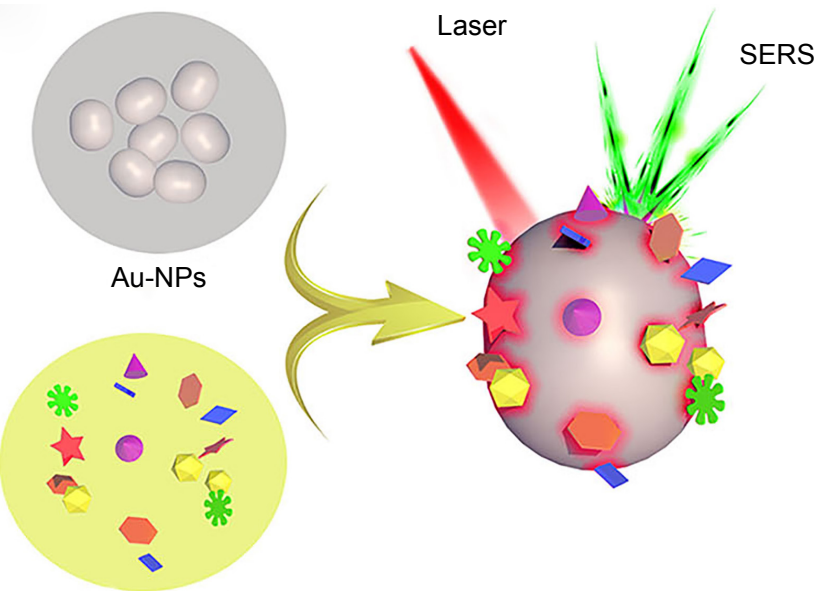

C

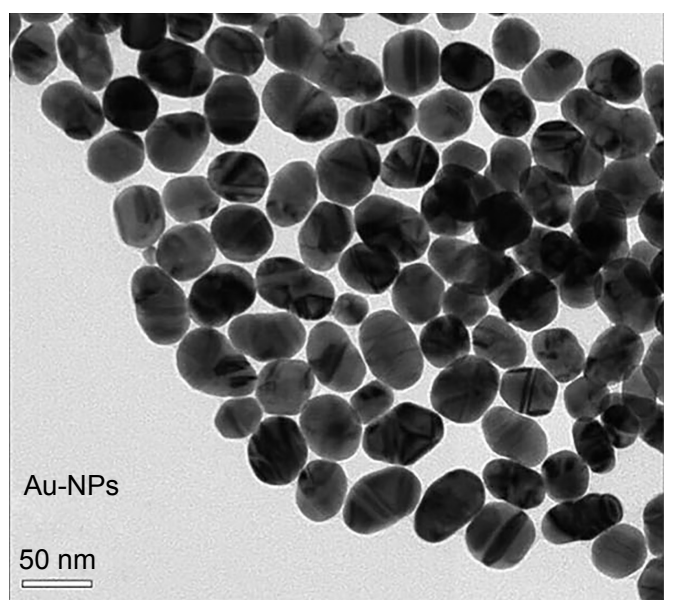

Molecular composition in plasma

Figure I (A) Schematic diagram of the SERS analysis of blood plasma. (B) The mechanism of SERS based on Au-NPs. (C) TEM image of Au-NPs. Note: The scale bars indicate $50 \mathrm{~nm}$.

Abbreviations: AU-NPs, gold nanoparticles; CCD, charge coupled device; SERS, surface-enhanced Raman spectroscopy; TEM, transmission electron microscopy.

for $88.9 \%$ of the variance to perform the LDA. As shown in Figure 4, the PCA-LDA model can clearly discriminate the plasma spectra of biochemical recurrence from bRFS. We applied leave-one-spectrum-out cross-validation method to validate the LDA discrimination model and revealed the diagnostic sensitivity, specificity, and accuracy of $65.8 \%$, $87.5 \%$, and $79.4 \%$, respectively. We used the ROC curve to evaluate the performance of clinical model CAPRA-S score, the Raman peak $1,328 \mathrm{~cm}^{-1}$, the Raman peak $1,328 \mathrm{~cm}^{-1}$ combined with CAPRA score, and PCA-LDA model based on SERS spectra. As shown in Figure 5, the AUC for the PCA-LDA model was 0.92 (0.86-0.97), the Raman peak $1,328 \mathrm{~cm}^{-1}$ was $0.73(0.62-0.84,95 \% \mathrm{CI})$, the CAPRA-S was $0.77(0.67-0.87,95 \% \mathrm{CI})$, and when combined Raman peak $1,328 \mathrm{~cm}^{-1}$ to CAPRA-S, the AUC value improved to $0.81(0.72-0.90,95 \% \mathrm{CI})$.

\section{Discussion}

It is valuable to identify patients who will develop early biochemical recurrence after RP because timely adjuvant therapy could improve their clinical outcomes., ${ }^{4,5}$ Previous studies indicated that blood chemical component information could be meaningful to supplement clinical risk stratification. ${ }^{12-14}$ In this study, we conducted overall analysis of comprehensive components in preoperative plasma using SERS technique and evaluated the value of corresponding Raman spectra for prediction of early biochemical recurrence (biochemical recurrence at $\leq 2$ years of $\mathrm{RP}$ ). Our results showed the 


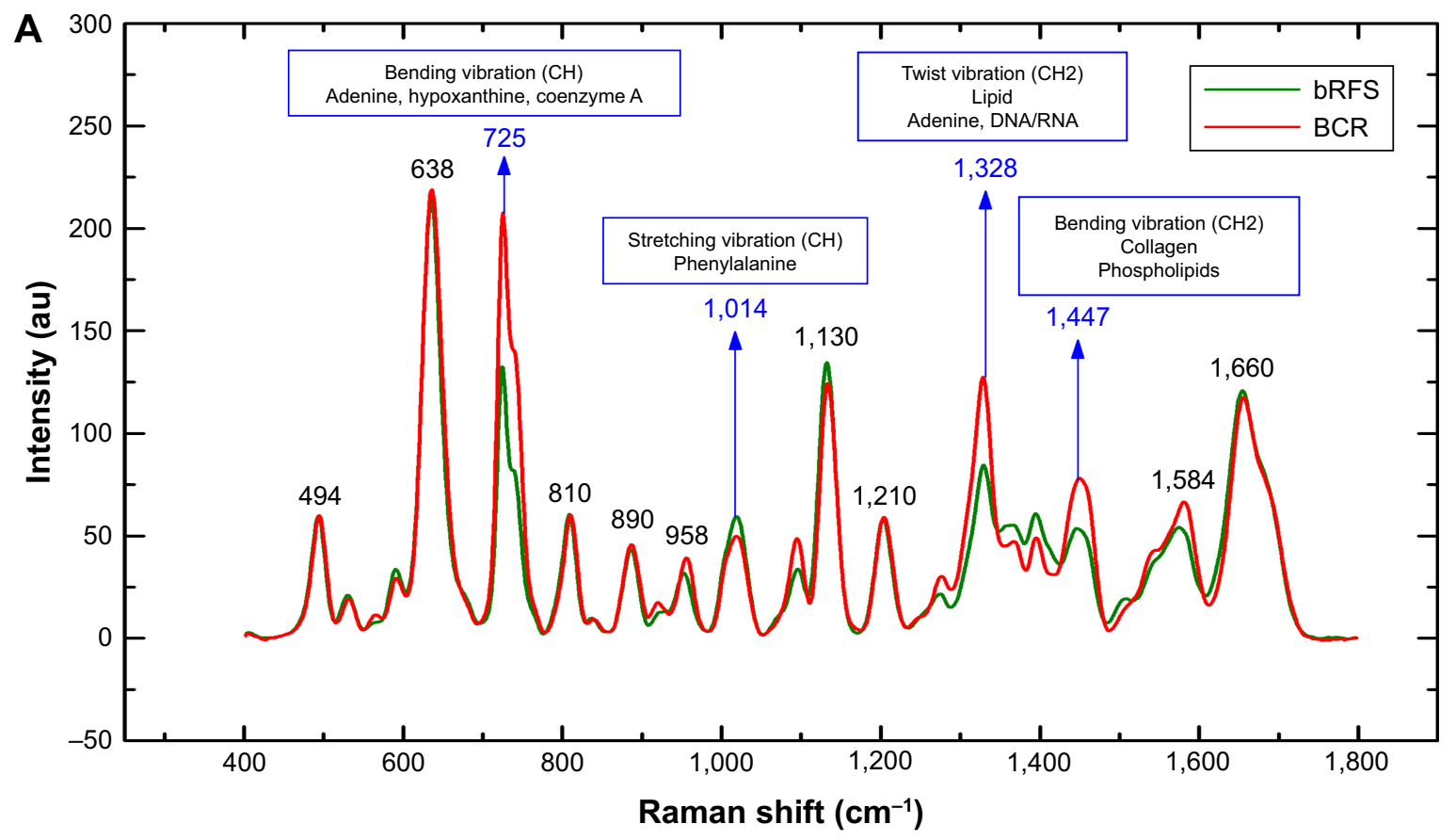

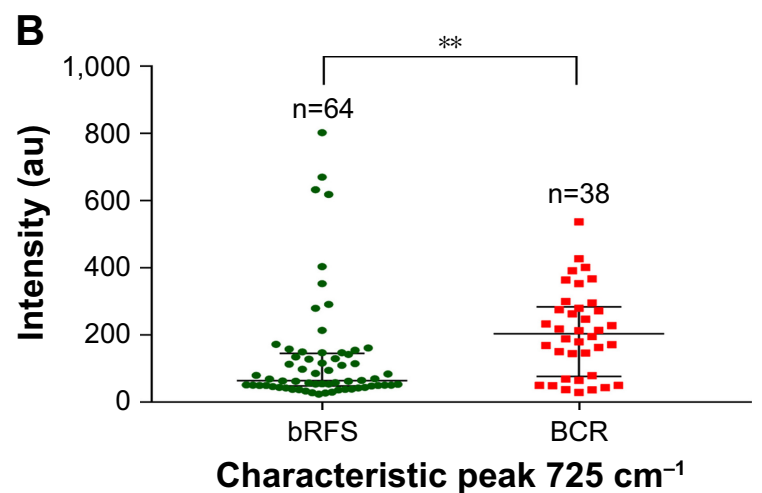

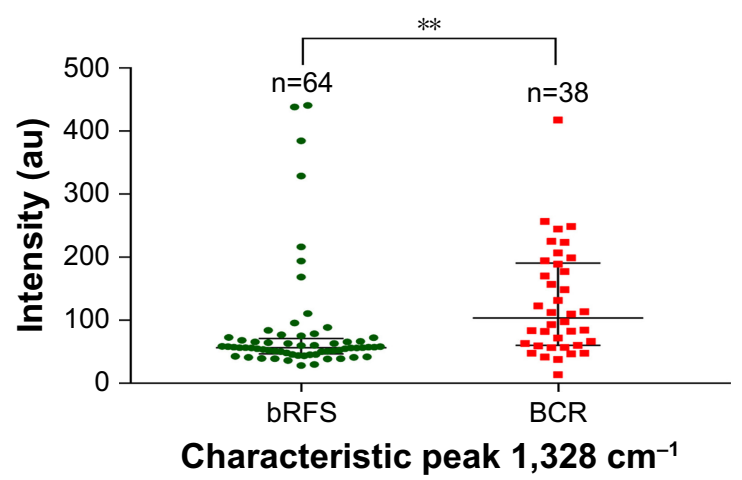

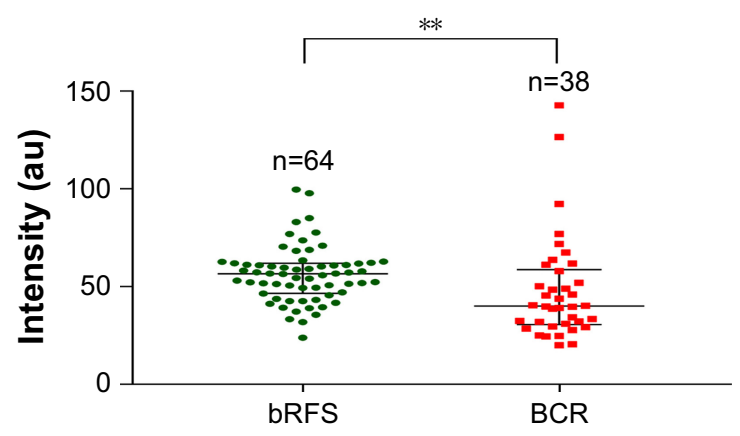

Characteristic peak $1,014 \mathrm{~cm}^{-1}$

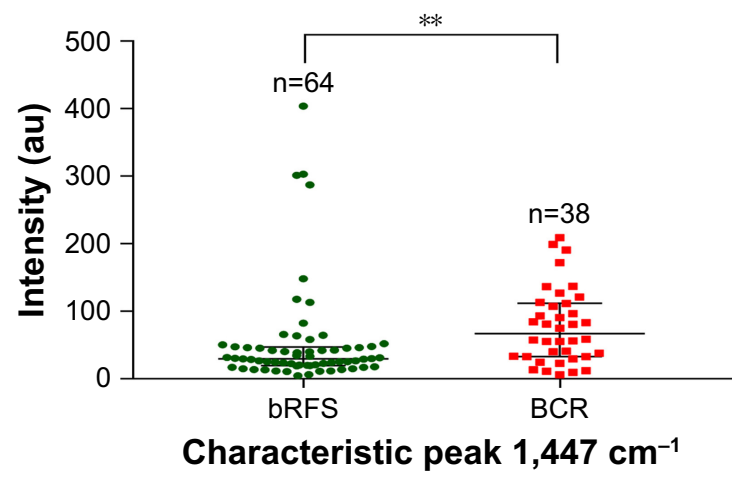

Figure 2 (A) Comparison of the normalized mean SERS spectra from 38 early biochemical recurrence and 64 bRFS plasma samples. (B) Box plots of the four significant SERS peak intensities $\left(725,1,014,1,328\right.$, and I,447 $\left.\mathrm{cm}^{-1}\right)$ for the two groups.

Notes: The line within each box represents the median, while the lower and upper boundaries of the box indicate first and third quartiles, respectively. Comparison of the spectra data via Mann-Whitney $U$ test, $* * P<0.01$.

Abbreviations: BCR, biochemical recurrence; bRFS, biochemical recurrence free survival; SERS, surface-enhanced Raman spectroscopy.

intensity of Raman peak $1,328 \mathrm{~cm}^{-1}$ was significantly associated with risk of early biochemical recurrence and could improve performance of CARPA-S scoring system for early biochemical recurrence prediction in patients treated by RP.
Raman spectroscopy (RS) is an optical technique relied on the energetic changes in inelastic light scattered from chemical bonds within the sample itself. Accordingly, the $\mathrm{RS}$ can provide native fingerprint information on the sample 
Table I Tentative assignments of significant SERS peaks observed in plasma ${ }^{17,19-23}$

\begin{tabular}{|c|c|c|}
\hline $\begin{array}{l}\text { Peak } \\
\text { position } / \mathbf{c m}^{-1}\end{array}$ & Vibrational mode & Assignment \\
\hline 638 & $\tau(C-S)$ & L-tyrosine, lactose \\
\hline 725 & $\mathrm{bv}(\mathrm{C}-\mathrm{H})$ & $\begin{array}{l}\text { Adenine, hypoxanthine, } \\
\text { coenzyme A }\end{array}$ \\
\hline 810 & $s(C-C-O)$ & L-serine \\
\hline 890 & $\delta(\mathrm{C}-\mathrm{O}-\mathrm{H})$ & Amino galactose \\
\hline 958 & $v(C-C)$ & Valine, proline \\
\hline 1,014 & $s(\mathrm{C}-\mathrm{H})$ & Phenylalanine \\
\hline 1,130 & $s(\mathrm{C}-\mathrm{N})$ & D-mannose \\
\hline 1,210 & $s(\mathrm{C}-\mathrm{N}), \mathrm{b} v(\mathrm{~N}-\mathrm{H})$ & Amide III \\
\hline 1,328 & $\tau\left(\mathrm{CH}_{2}\right)$ & $\begin{array}{l}\text { Lipids, } \mathrm{CH} \text { vibration in } \\
\text { DNA/RNA }\end{array}$ \\
\hline $\mathrm{I}, 447$ & $\mathrm{bv}\left(\mathrm{C}-\mathrm{H}_{2}\right)$ & Collagen, phospholipids \\
\hline ।,584 & $\mathrm{bv}(\mathrm{C}=\mathrm{C})$ & Acetoacetate \\
\hline 1,660 & $s(C=C):(\beta$-pleated sheet $)$ & Amide I \\
\hline
\end{tabular}

Abbreviations: $v$, vibration; $\tau$, twist vibration; bv, bending vibration; s, stretching vibration; $\delta$, scissoring vibration; SERS, surface-enhanced Raman spectroscopy.

determined by the constituents and the environment, which have been successfully used in discriminating benign from cancer tissue in other malignancies such as breast cancer, kidney cancer, and PCa. ${ }^{17,18}$ SERS combined RS with metallic nano-substrate, which can achieve $10^{6}-10^{8}$ times of Raman signal enhancement caused by electromagnetic effect and enable collection of high quality Raman spectra signal. ${ }^{19}$ SERS is suitable for bio-sample analysis such as blood, secretions, and saliva for its advantages of quick response, high accuracy and sensitivity, and slight samples requirement, which have been used to detect nasopharyngeal, gastric, cervical, and hepatocellular cancers by analyzing blood. ${ }^{20-23}$ Our group also reported value of SERS in early detection of PCa by analyzing components of serum and expressed prostatic secretion. ${ }^{24}$

In this study, significantly stronger intensities at Raman spectral peaks $725,1,328$, and $1,447 \mathrm{~cm}^{-1}$, and lower intensities at $1,014 \mathrm{~cm}^{-1}(P<0.01)$ were identified in the early biochemical recurrence group compared to bRFS group.

Table 2 Univariate and multivariate Cox proportional hazard analyses of Raman peaks and CAPRA-S

\begin{tabular}{|l|l|l|l|}
\hline Model & Variable & $\begin{array}{l}\text { HR } \\
\mathbf{( 9 5 \% ~ C I )}\end{array}$ & P-value \\
\hline \multirow{3}{*}{ Univariate } & CAPRA-S & $1.67(\mathrm{I} .37-2.03)$ & $<0.00 \mathrm{I}$ \\
\cline { 2 - 4 } & $\begin{array}{l}\text { Peak I,328 } \mathrm{cm}^{-1} \\
\text { (increase by a quarter) }\end{array}$ & $1.97(\mathrm{I} .4 \mathrm{I}-2.74)$ & $<0.00 \mathrm{I}$ \\
\hline Multivariate & CAPRA-S & $1.60(\mathrm{I} .32-1.95)$ & $<0.00 \mathrm{I}$ \\
\cline { 2 - 4 } & $\begin{array}{l}\text { Peak I,328 } \mathrm{cm}^{-1} \\
\text { (increase by a quarter) }\end{array}$ & $1.67(\mathrm{I} .19-2.33)$ & 0.003 \\
\hline
\end{tabular}

Abbreviation: CAPRA-S, Cancer of the Prostate Risk Assessment postsurgical score.

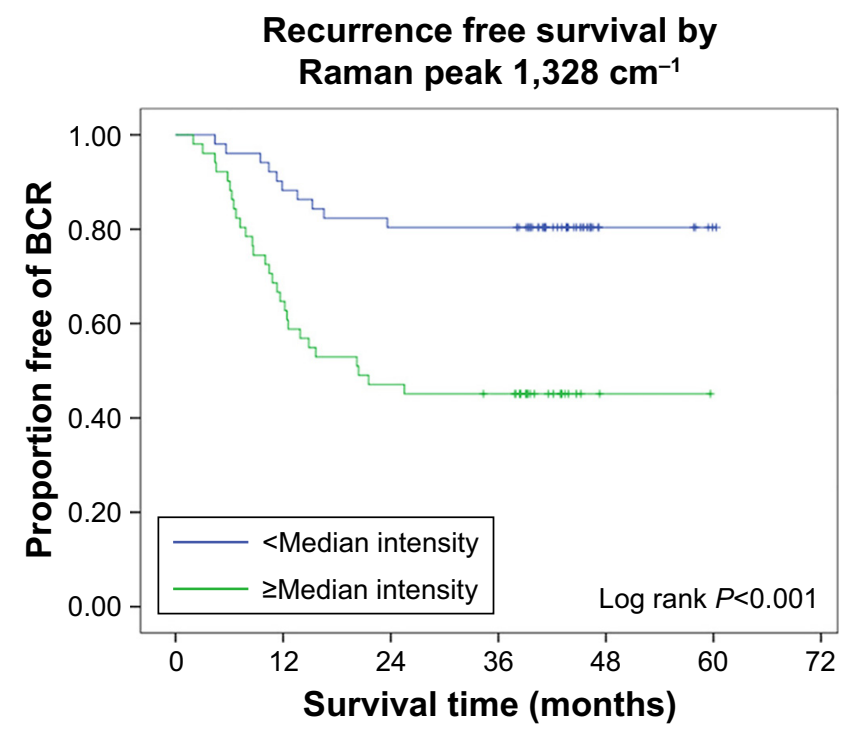

Number at risk

\begin{tabular}{|l|l|l|l|l|l|}
\hline Time (months) & 0 & 12 & 24 & 36 & 48 \\
\hline <Median intensity & 51 & 45 & 41 & 40 & 5 \\
\hline$\geq$ Median intensity & 51 & 32 & 23 & 21 & 3 \\
\hline
\end{tabular}

Figure 3 Kaplan-Meier curve showing association of Raman peak $1,328 \mathrm{~cm}^{-1}$ and risk of early biochemical recurrence.

Abbreviation: $\mathrm{BCR}$, biochemical recurrence.

According to the tentative biochemical assignments, blood of early biochemical recurrence patients might contain higher concentrations of nucleic acid bases like adenine, hypoxanthine, DNA/RNA bases (725 and 1,328 $\mathrm{cm}^{-1}$ ), a group

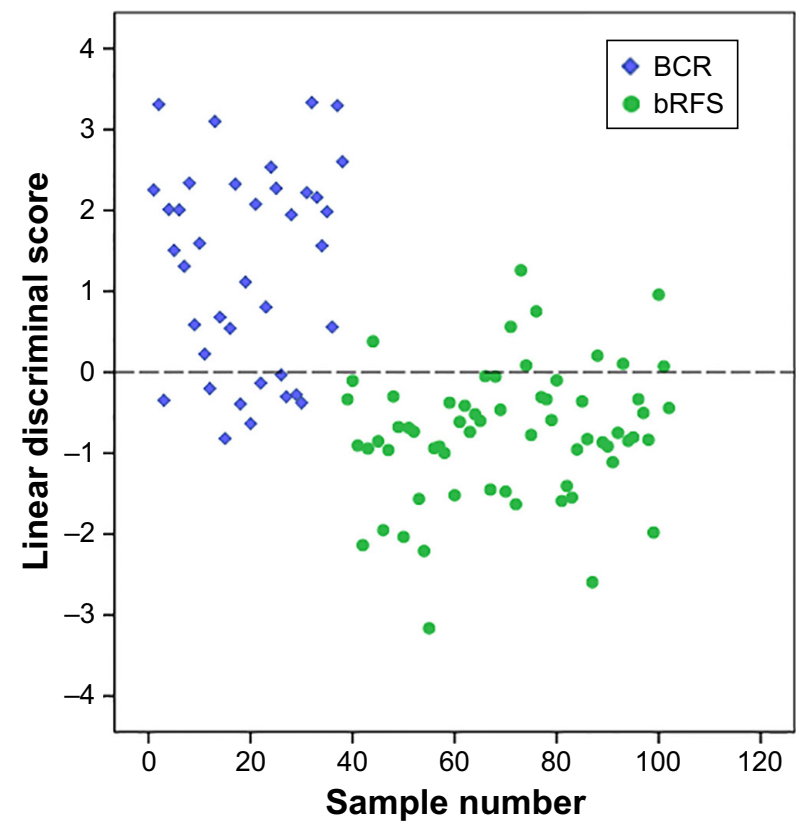

Figure 4 PCA and scatter plots of LDA score of biochemical recurrence and bRFS plasma SERS spectra.

Abbreviations: $B C R$, biochemical recurrence; $b R F S$, biochemical recurrence free survival; LDA, linear discriminant analysis; PCA, principal component analysis; SERS, surface-enhanced Raman spectroscopy. 


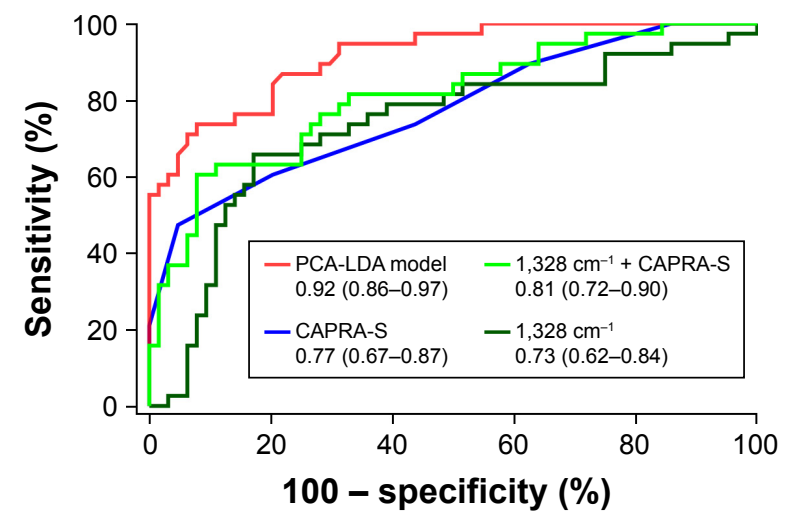

Figure 5 Comparison of ROC curves of PCA-LDA model, Raman peak $1,328 \mathrm{~cm}^{-1}$ combined with CAPRA-S score, CAPRA-S score alone, and Raman peak $1,328 \mathrm{~cm}^{-1}$ alone.

Abbreviations: CAPRA-S, Cancer of the Prostate Risk Assessment postsurgical score; PCA-LDA, principal component analysis and linear discriminate analysis; ROC, receiver operating characteristic.

molecules of lipid metabolism like coenzyme A $\left(725 \mathrm{~cm}^{-1}\right)$, lipids $\left(1,328 \mathrm{~cm}^{-1}\right)$, and phospholipids $\left(1,447 \mathrm{~cm}^{-1}\right)$. It should be explained that the same vibrational bond can be distributed in many other molecules, so that the individual Raman spectral peak could be a mixture of different molecules. Previous studies have reported that PCa patients had higher concentration of cell-free DNA in plasma as compared to men with benign prostatic hyperplasia. ${ }^{25,26}$ In addition, elevated blood cell-free DNA concentration was significantly associated with increased risk of biochemical recurrence within 2 years of RP, indicating more aggressive nature of cancer. ${ }^{27,28}$ The increased nucleic acid bases mostly originated from the increased tumor cell proliferation rate, which was considered as an important parameter for cancer detection and monitoring. ${ }^{29}$ However, several recent studies reported that the detection of circulating tumor cells did not correlate significantly with clinical characteristics, or the development of biochemical recurrence. Of course, larger cohorts with extended follow-up are needed to validate these findings. In our study, although the SERS spectra could only provide rough information of nucleic acid bases, like concentrations of adenine, hypoxanthine, rather than determined aberrant tumor DNA sequences, the Raman spectral peaks corresponded well to the entire change of blood nucleic acid bases. It was recognized that time to biochemical recurrence may be closely associated with the risk of clinical metastases, ${ }^{4,5}$ hence we speculated that those Raman spectral features in our study might be emblem of occult metastases in patients who developed early biochemical recurrence. In other words, the distinct Raman spectral peaks 725 (coenzyme A), 1,328 (lipids), and 1,447 $\mathrm{cm}^{-1}$ (phospholipids) might also result from dysregulation of lipid metabolism, such as increased lipid accumulation, upregulation of several lipogenic enzymes (coenzyme A), and dysregulation of phospholipid metabolism. ${ }^{30}$ SERS peak at $1,014 \mathrm{~cm}^{-1}$ was found lower in biochemical recurrence plasma as compared to bRFS, indicating a decrease in the percentage of phenylalanine relative to the total SERS-active molecular composition. Decrease in Raman signals of phenylalanine were also observed in nasopharyngeal cancer, blood, and gastric malignancy tissues, ${ }^{19,20}$ suggesting that the phenylalanine metabolism was probably associated with tumor formation and progression.

Since the SERS was comprehensive for sample analysis, the Raman spectra of plasma mostly reflected rough and mixed information of various components in blood. The value of distinct Raman peaks for early biochemical recurrence prediction was evaluated and $1,328 \mathrm{~cm}^{-1}$ was considered valuable for early biochemical recurrence prediction. The typical Raman spectral peak $1,328 \mathrm{~cm}^{-1}$ was associated with significant risk of early biochemical recurrence in univariate and multivariate Cox regression models and stronger intensity of Raman indicated higher risk of early biochemical recurrence. Kaplan-Meier curve showed patients with high intensity ( $\geq$ median intensity) in Raman peak $1,328 \mathrm{~cm}^{-1}$ were more likely to develop early biochemical recurrence within 2 years. These results indicated that Raman peak $1,328 \mathrm{~cm}^{-1}$ could be an independent risk factor for early biochemical recurrence. The CAPRA-S score was considered as performing tool for recurrence prediction after RP, which was well validated in the Shared Equal Access Regional Cancer Hospital database, which included a large, multi-institutional cohort of patients. ${ }^{8}$ The CAPRA-S score incorporated all important risk factors including PSA, Gleason score, positive surgical margin, seminal vesicle invasion, and T-stage. The clinical characters in our study were consistent with previous studies. The early biochemical recurrence group contained significantly higher proportion of high risk (6-10) CAPRA-S than bRFS group (47\% vs 3\%). The CAPRA-S and Raman peak $1,328 \mathrm{~cm}^{-1}$ were both significantly independent predictor of early biochemical recurrence. Although the Raman peak $1,328 \mathrm{~cm}^{-1}$ did not perform better than CAPRA-S alone for early biochemical recurrence prediction, discrimination was better when combined them together. In addition, PCA-LDA model based on SERS spectra showed good ability of discriminating early biochemical recurrence from bRFS patients, and then was validated by the leaveone-spectrum-out cross-validation method, which revealed the diagnostic sensitivity, specificity, and accuracy of $65.8 \%$, $87.5 \%$, and $79.4 \%$, respectively.

Several limitations for our study should be stated. First, the study was conducted in a single center and the sample 
size was relatively small. To reduce variation, we collected three SERS spectra from different regions of each sample for data analysis, and the results demonstrated that the Raman spectra between two groups were significantly different. Further study with larger sample number would be desirable to validate our results. Second, the component changes reflected by SERS were on the basis of tentative assignments in published documents and RS database, which may be confirmed by other methods in future studies. Despite those limitations, our study firstly reported the promising results of SERS analysis of serum components for the prediction of early biochemical recurrence, which might be meaningful for developing individual treatment strategies. Our preliminary study might promote the application of SERS in medicine.

\section{Conclusion}

In conclusion, in this preliminary study, we have reported the value of SERS analysis of preoperative plasma to identify patients with high risk of early biochemical recurrence (at $\leq 2$ years of RP) after RP. The addition of distinct SERS spectra could improve the power of classic clinical model for early biochemical recurrence prediction in $\mathrm{PCa}$ patients.

\section{Acknowledgments}

This study was supported by National Natural Science Foundation of China (81572536, 81672850), Science and Technology Commission of Shanghai Municipality (14140901700, 16411969800), the Joint Research Foundation for Innovative Medical Technology of Shanghai Shenkang Hospital Development Center (SHDC12015125), Shanghai Municipal Education Commission (15ZZ058), Shanghai Municipal Commission of Health and Family Planning (201640247), Shanghai Municipal Education Commission-Gaofeng Clinical Medicine Grant Support (20152215), Key Disciplines Group Construction Project of Pudong Health Bureau of Shanghai (PWZxq2014-05), and Innovation Fund for Translational Research of Shanghai Jiao Tong University School of Medicine (15ZH4002).

\section{Disclosure}

The authors report no conflicts of interest in this work.

\section{References}

1. Siegel RL, Miller KD, Jemal A. Cancer statistics, 2016. CA Cancer J Clin. 2016;66(1):7-30.

2. Novara G, Ficarra V, Mocellin S, et al. Systematic review and metaanalysis of studies reporting oncologic outcome after robot-assisted radical prostatectomy. Eur Urol. 2012;62(3):382-404.

3. Freedland SJ, Humphreys EB, Mangold LA, et al. Risk of prostate cancer-specific mortality following biochemical recurrence after radical prostatectomy. JAMA. 2005;294(4):433-439.
4. Fossati N, Karnes RJ, Cozzarini C, et al. Assessing the optimal timing for early salvage radiation therapy in patients with prostate-specific antigen rise after radical prostatectomy. Eur Urol. 2016;69(4):728-733.

5. Abugharib A, Jackson WC, Tumati V, et al. Very early salvage radiotherapy improves distant metastasis-free survival. J Urol. 2017; 197(3):662-668.

6. Messing EM, Manola J, Yao J, et al. Immediate versus deferred androgen deprivation treatment in patients with node-positive prostate cancer after radical prostatectomy and pelvic lymphadenectomy. Lancet Oncol. 2006;7(6):472-479.

7. Tilki D, Mandel P, Schlomm T, et al. External validation of the CAPRA-S score to predict biochemical recurrence, metastasis and mortality after radical prostatectomy in a European cohort. J Urol. 2015;193(6): 1970-1975.

8. Punnen S, Freedland SJ, Presti JC, et al. Multi-institutional validation of the CAPRA-S score to predict disease recurrence and mortality after radical prostatectomy. Eur Urol. 2014;65(6):1171-1177.

9. Walz J, Chun FK, Klein EA, et al. Nomogram predicting the probability of early recurrence after radical prostatectomy for prostate cancer. J Urol. 2009;181(2):601-608.

10. Hansen J, Bianchi M, Sun M, et al. Percentage of high-grade tumour volume does not meaningfully improve prediction of early biochemical recurrence after radical prostatectomy compared with Gleason score. BJU Int. 2014;113(3):399-407.

11. Kelly RS, Vander Heiden MG, Giovannucci E, Mucci LA. Metabolomic biomarkers of prostate cancer: prediction, diagnosis, progression, prognosis, and recurrence. Cancer Epidemiol Biomarkers Prev. 2016; 25(6):887-906.

12. Maxeiner A, Adkins CB, Zhang Y, et al. Retrospective analysis of prostate cancer recurrence potential with tissue metabolomic profiles. Prostate. 2010;70(7):710-717.

13. Mcdunn JE, Li Z, Adam KP, et al. Metabolomic signatures of aggressive prostate cancer. Prostate. 2013;73(14):1547-1560.

14. Stabler S, Koyama T, Zhao Z, et al. Serum methionine metabolites are risk factors for metastatic prostate cancer progression. PLoS One. 2011;6(8):e22486.

15. Lane LA, Qian X, Nie S. SERS nanoparticles in medicine: from labelfree detection to spectroscopic tagging. Chem Rev. 2015;115(19): 10489-10529.

16. Bastús NG, Comenge J, Puntes V, Puntes V. Kinetically controlled seeded growth synthesis of citrate-stabilized gold nanoparticles of up to $200 \mathrm{~nm}$ : size focusing versus Ostwald ripening. Langmuir. 2011; 27(17):11098-11105.

17. Jermyn M, Desroches J, Aubertin K, et al. A review of Raman spectroscopy advances with an emphasis on clinical translation challenges in oncology. Phys Med Biol. 2016;61(23):R370-R400.

18. Aubertin K, Trinh VQ, Jermyn M, et al. Mesoscopic characterization of prostate cancer using Raman spectroscopy: potential for diagnostics and therapeutics. BJU Int. 2018;122(2):326-336.

19. Schlücker S. Surface-enhanced Raman spectroscopy: concepts and chemical applications. Angew Chem Int Ed Engl. 2014;53(19): 4756-4795.

20. Feng S, Chen R, Lin J, et al. Nasopharyngeal cancer detection based on blood plasma surface-enhanced Raman spectroscopy and multivariate analysis. Biosens Bioelectron. 2010;25(11):2414-2419.

21. Feng S, Chen R, Lin J, et al. Gastric cancer detection based on blood plasma surface-enhanced Raman spectroscopy excited by polarized laser light. Biosens Bioelectron. 2011;26(7):3167-3174.

22. Feng S, Lin D, Lin J, et al. Blood plasma surface-enhanced Raman spectroscopy for non-invasive optical detection of cervical cancer. Analyst. 2013;138(14):3967-3974.

23. Xiao R, Zhang X, Rong Z, et al. Non-invasive detection of hepatocellular carcinoma serum metabolic profile through surface-enhanced Raman spectroscopy. Nanomedicine. 2016;12(8):2475-2484.

24. Shao X, Pan J, Wang Y, et al. Evaluation of expressed prostatic secretion and serum using surface-enhanced Raman spectroscopy for the noninvasive detection of prostate cancer, a preliminary study. Nanomedicine. 2017;13(3):1051-1059. 
25. Feng J, Gang F, Li X, et al. Plasma cell-free DNA and its DNA integrity as biomarker to distinguish prostate cancer from benign prostatic hyperplasia in patients with increased serum prostate-specific antigen. Int Urol Nephrol. 2013;45(4):1023-1028.

26. Yin C, Luo C, Hu W, Ding X, Yuan C, Wang F. Quantitative and qualitative analysis of circulating cell-free dna can be used as an adjuvant tool for prostate cancer screening: a meta-analysis. Disease Markers. 2016;2016(4):1-12.

27. Bastian PJ, Palapattu GS, Yegnasubramanian S, et al. Prognostic value of preoperative serum cell-free circulating DNA in men with prostate cancer undergoing radical prostatectomy. Clin Cancer Res. 2007; 13(18 Pt 1):5361-5367.
28. Wroclawski ML, Serpa-Neto A, Fonseca FL, et al. Cell-free plasma DNA as biochemical biomarker for the diagnosis and follow-up of prostate cancer patients. Tumour Biol. 2013;34(5):2921-2927.

29. Di Meo A, Bartlett J, Cheng Y, Pasic MD, Yousef GM. Liquid biopsy: a step forward towards precision medicine in urologic malignancies. Mol Cancer. 2017;16(1):80.

30. Wu X, Daniels G, Lee P, Monaco ME. Lipid metabolism in prostate cancer. Am J Clin Exp Urol. 2014;2(2):111-120. 


\section{Supplementary material}

Table SI Clinical and pathologic information of the study subjects

\begin{tabular}{|c|c|c|c|}
\hline Clinical characteristics & bRFS & BCR & Two-tailed $P$-value \\
\hline Total, N & 64 & 38 & \\
\hline Age, years, mean (SD) & $67.9(6.2)$ & $67.1(6.3)$ & 0.505 \\
\hline PSA, ng/mL, median (IQR) & $11.6(7.8)$ & $18.8(23.9)$ & 0.001 \\
\hline Pathologic Gleason score, n (\%) & & & 0.011 \\
\hline$<7 / 3+4$ & $37(57.8)$ & $13(34.2)$ & \\
\hline $4+3$ & $16(25)$ & $9(23.7)$ & \\
\hline $8-10$ & $11(17.2)$ & $16(42.1)$ & \\
\hline Extracapsular extension, n (\%) & $3(4.6)$ & $6(15.8)$ & 0.056 \\
\hline Infiltration of seminal vesicles, $\mathrm{n}(\%)$ & $3(4.6)$ & $6(15.8)$ & 0.056 \\
\hline Positive surgical margins, n (\%) & $3(4.6)$ & $9(23.7)$ & 0.004 \\
\hline Lymph node invasion, $\mathrm{n}(\%)$ & 0 & $\mathrm{I}(2.6)$ & 0.192 \\
\hline Clinical stage, n (\%) & & & 0.019 \\
\hline $\mathrm{p} \mathrm{T}<3$ & $56(87.5)$ & $26(68.4)$ & \\
\hline $\mathrm{pT} \geq 3$ & $8(12.5)$ & $12(31.6)$ & \\
\hline CAPRA score, median (IQR) & $3(2)$ & $5(3)$ & \\
\hline CAPRA score, n (\%) & & & $<0.001$ \\
\hline $0-2$ & $24(37.5)$ & $4(10.5)$ & \\
\hline $3-5$ & $37(57.8)$ & $16(42.1)$ & \\
\hline $6-10$ & $3(4.7)$ & $18(47.4)$ & \\
\hline
\end{tabular}

Abbreviations: BCR, biochemical recurrence; bRFS, biochemical recurrence free survival; CAPRA, Cancer of the Prostate Risk Assessment; pT, pathological stage.

\section{Publish your work in this journal}

The International Journal of Nanomedicine is an international, peerreviewed journal focusing on the application of nanotechnology in diagnostics, therapeutics, and drug delivery systems throughout the biomedical field. This journal is indexed on PubMed Central, MedLine, CAS, SciSearch $®$, Current Contents ${ }^{\circledR} /$ Clinical Medicine,
Journal Citation Reports/Science Edition, EMBase, Scopus and the Elsevier Bibliographic databases. The manuscript management system is completely online and includes a very quick and fair peer-review system, which is all easy to use. Visit http://www.dovepress.com/ testimonials.php to read real quotes from published authors. 